\title{
EVOLUCIÓN DEL PERFIL SOCIOACADÉMICO DE LA FÍSICA EN ESTUDIANTES VENEZOLANOS
}

\author{
SEBASTIÁ, JOSÉ M. ${ }^{1}$ y MONCADA, PABLO² \\ ${ }^{1}$ Departamento de Física. Universidad Simón Bolívar. Apartado 89.000. Caracas 1080-A. Venezuela. \\ ${ }^{2}$ Centro Nacional para el Mejoramiento de la Enseñanza de las Ciencias, CENAMEC. \\ Apartado 75.055. Caracas 1070-A. Venezuela.
}

\begin{abstract}
SUMMARY
This work presents a comparative and descriptive analisys of the socio-academic profile shown by students of physics in Venezuela, and the relationship between this profile and variables as: educational level, gender, academic performance in physics, and general attitude towards science. Our results, from a $825 \mathrm{High}$ School sample, reveals a decrease in the socio-academic profile of subject and attitude along the school year. However, no decrease was found between a given school level and the next. The subject profile obtained from male student scores was always higher than the corresponding one obtained from female student scores. Their evolutions were similar, with an increase in the gap between the two as the educational level increased.
\end{abstract}

\section{INTRODUCCIÓN}

Las opiniones y actitudes que los estudiantes tienen hacia la ciencia, en general, y hacia alguna asignatura, en particular, no son innatas ni son inmutables. Se desarrollan en el contexto cotidiano y evolucionan en la interacción del estudiante con su entorno particular, tornándose más positivas o más negativas, dependiendo de múltiples factores (Gardner, 1975; Kuhn, 1979; Shrigley, 1983; Escudero y Lacasta, 1984; Solomon, 1987).

La inclusión de las actitudes en las metas currriculares ha sido siempre un tema controversial. Algunos defienden la necesidad de incluir las actitudes entre las metas curriculares debido a que suponen que las actitudes poseen un valor en sí mismas, ya que pueden ser consideradas tanto o más importantes que el aprendizaje de conocimientos específicos. Otros fomentan la inclusión de objetivos actitudinales en el currículo, en el supuesto de que una actitud positiva hacia una asignatura facilita- ría el aprendizaje de la misma. Por el contrario, algunos esgrimen argumentos en contra de su inclusión, ya que la enseñanza de actitudes podría ser considerada como un adoctrinamiento a los estudiantes. El hecho cierto es que el área de las actitudes sigue siendo un área periférica y marginal en los diseños curriculares y que se carece de las suficientes investigaciones para justificar su inclusión o exclusión en el currículo (Kozlow y Nay, 1976; Peterson y Carlson, 1979; Fraser, 1982; Schibeci, 1989; Koballa, 1992).

Nuestro estudio tiene como objetivo lograr una mejor comprensión de los factores que afectan la actitud de los estudiantes hacia una área del conocimiento en particular: la física. Para ello, analizamos la percepción que los estudiantes tienen de la misma y la evolución de dicha percepción en relación con el nivel educativo, el género (masculino-femenino) del estudiante y las notas obteni- 
das en la asignatura de física. Asimismo, analizamos la vinculación existente entre la percepción socioacadémica de la física y la actitud general del estudiante hacia la ciencia.

\section{REVISIÓN BIBLIOGRÁFICA}

La mayoría de los estudios en el área han sido generados por la suposición de que las actitudes y el aprendizaje estarían fuertemente relacionados y que, por lo tanto, una mejora en las actitudes se traduciría en una mejora del rendimiento del estudiante. Sin embargo, los resultados que han sido reportados hasta ahora revelan que, aunque parece existir relación entre actitudes y rendimiento, esta asociación es débil (Malone y Fleming, 1983). En opinión de Fraser (1982), si los profesores desean aumentar el rendimiento, deben concentrarse en mejorar el rendimiento per se, en lugar de intentar mejorar las actitudes. Además, no es claro si son las actitudes positivas las que favorecen el rendimiento o si los estudiantes que tiene buen rendimiento adoptan actitudes positivas hacia la asignatura, aunque parece ser que la relación actitudes-rendimiento es bidireccional (Schibeci y Riley,1986). Algunos autores (Hasan, 1985; Oliver y Simpson, 1988) han señalado que las relaciones más fuertes entre actitud y aprendizaje se producen a través de otra variable: el autoconcepto que tiene el estudiante de su capacidad para la ciencia (self concept of achievement in science), en el sentido de estar convencido de ser capaz de tener éxito en ciencias.

En relación con la evolución de las opiniones y actitudes de los estudiantes con la edad, el nivel educativo y los cursos de ciencias, Yager y Penick (1984) encontraron que la ciencia era descrita como atractiva, interesante y útil, por los estudiantes de la escuela elemental, pero ocurría una fuerte declinación en las opiniones favorables cuando estos estudiantes llegaban a la escuela superior.

En un estudio longitudinal llevado cabo por Kelly (1986) con una muestra de 1.300 estudiantes de diez escuelas, las opiniones positivas de los estudiantes hacia la ciencia se tornaban más negativas entre los 12 y los 14 años. En un estudio realizado en Alemania con una muestra de más de 4.000 estudiantes entre los 11 y los 16 años, Haussler (1987) pudo determinar que las actitudes hacia la ciencias y las materias científicas tienen una fuerte declinación entre los 12 y 13 años cuando los estudiantes comienzan a estudiar la asignatura de física. Algunos han sugerido que esta declinación puede estar relacionada con un aumento de aversión hacia la escuela en general, aunque se ha detectado que es mayor en las asignaturas de ciencias que en las otras materias.

En cuanto a la modificación de actitudes de los estudiantes con el número de cursos de ciencias, algunos resultados indican una declinación de la actitud favorable hacia la ciencia al aumentar el número de cursos de ciencias seguidos por el estudiante (Yager y Penick, 1986). Esta erosión de la actitud hacia la ciencia ha sido posible determinarla incluso a lo largo del mismo año escolar (Cannon y Simpson, 1985). Por el contrario, Gogolin y Swartz (1992) detectaron un cambio de actitudes favorable a la ciencia con la instrucción en cursos de ciencias. Los resultados mostraron diferencias significativas entre ambos grupos para seis variables analizadas en los resultados del test ATSI (Attitude Toward Science Inventory). Sus resultados sugieren que la actitud hacia la ciencia mejora con la exposición a cursos de ciencias, pero parece depender mucho de la calidad de dichos cursos. Por lo tanto, los resultados de las relaciones de la actitud con la instrucción son mixtos: algunos estudios indican que el número de cursos de ciencias tiene un efecto acumulativo positivo (Gabel, 1981; Gogolin y Swartz, 1992) mientras otros señalan que la influencia es claramente negativa (Gardner, 1975; Sadava, 1976).

La variable aislada que ha mostrado más correlación con la actitud es el género de los estudiantes: los varones tienen en su mayoría una actitud más favorable hacia la ciencia que las mujeres (Hamilton, 1982; Cannon y Simpson, 1985) y esta posición es compartida por los profesores y profesoras (Fraser-Abder y Shrigley, 1980). Sin embargo, esta proporción se invierte en las materias de biología y química (Hofstein, Ben-Zvi, Samuel, y Tamir, 1977). Según los resultados existentes, cuando existe declinación en las actitudes, ésta es similar en las muchachas y en los muchachos, aunque tanto Hofstein, Ben-Zvi, Samuel (1976) como Haussler (1987) encontraron que las diferencias de actitud entre ambos géneros van disminuyendo con la edad.

La relación de la actitud con el nivel socioeconómico (SES, Socio Economic Status) no ha sido estudiada en profundidad. En un estudio exploratorio llevado a cabo en Venezuela, Moncada y Sebastiá (1986) detectaron una actitud ligeramente más favorable hacia la ciencia en estudiantes de las clases bajas de la población que en estudiantes de las clases acomodadas. Sin embargo, Kelly (1986) detectó que los estudiantes de clase media tenían una actitud ligeramente más positiva que los de clase baja (determinada fundamentalmente por la profesión de los padres). En otros estudios (Fraser, 1982; Hadden y Johnstone, 1983) no se reportó asociación significativa entre el SES y cualquiera de las variables de actitud hacia la ciencia.

\section{PROPÓSITOS DE LA INVESTIGACIÓN}

La presente investigación tuvo como propósitos:

1) Realizar una descripción cuantitativa de la variable Perfil de la física a partir de las opiniones de los estudiantes del bachillerato venezolano en cuanto a su valor académico intrínseco (interesante, comprensible, fácil, etc.), y en cuanto a su implicación social (útil, beneficiosa, segura, etc.), y analizar la evolución que tiene dicho perfil con el nivel educativo y el subnivel educativo (trimestre). 
2) Realizar un estudio comparativo de la variable Perfil de la física en función de: niveles educativos, trimestre de estudio, género (masculino, femenino) de los estudiantes y notas obtenidas en la asignatura de física.

3) Realizar un estudio comparativo y asociativo entre la evolución de la variable Perfil de la física y la variable Actitud general de los estudiantes hacia la ciencia, analizando las influencias de ambas.

\section{METODOLOGÍA: PROCEDIMIENTO, MUES- TRA E INSTRUMENTO}

\section{Procedimiento}

Se llevó a cabo un análisis descriptivo, comparativo y asociativo, de nueve grupos (tres niveles educativos y tres subniveles o trimestres). Las variables dependientes del estudio fueron: $a$ ) perfil socioacadémico, dividido en dos subvariables: perfil social y perfil académico; b) actitud general hacia la ciencia. Las variables independientes del estudio fueron: $a$ ) nivel educativo; b) lapso académico (trimestre); c) género del estudiante; y d) nota de la asignatura de Física.

Los datos fueron recolectados en momentos próximos a la finalización del lapso académico (trimestre), ya que se pretendía detectar el efecto que el curso tenía sobre la percepción del estudiante de la física y sobre la actitud del estudiante hacia la ciencia en general. La recolección de datos se efectuó en las clases habituales de la asignatura y fue llevada a cabo por el profesor de la misma.

\section{Muestra \\ La muestra de estudiantes analizada estuvo constituida por 825 estudiantes (430 varones, 52\%, y 395 mujeres, $48 \%$ ) de los tres niveles en los que se estudia física en Venezuela. En el primer nivel (noveno grado de escuela básica), la muestra estuvo conformada por 135 estudian- tes en el primer trimestre, 123 en el segundo y 123 en el tercero; la edad más frecuente en ese nivel era de 15 años (media, 14,54; mediana, 15; moda, 15). En el segundo nivel (primer año de educación media diversificada), la muestra estuvo constituida por 81 estudiantes el primer trimestre, 87 el segundo y 84 el tercero; la edad más frecuente era 16 años (media, 15,5; mediana, 16; moda, 16). En el tercer nivel (segundo año de educación media diversificada), la muestra estuvo constituida por 56 estudiantes el primer trimestre, 69 el segundo y 67 el tercero; la edad más frecuente en ese nivel era de 17 años (media,16,5; mediana, 17; moda 17).}

\section{Instrumentos}

Existe una amplia batería de tests desarrollados para detectar actitudes cuya validez y confiabilidad ha sido suficientemente determinada (Osgood, 1969; Fraser, 1977; Schibeci, 1982). Sin embargo, estos instrumentos desarrollados y validados en inglés son difíciles de adaptar a poblaciones cuya primera lengua no es la inglesa, ya que los instrumentos que pretenden medir actitudes parecen ser más sensibles a la formulación verbal que los tests de conocimientos (Moreno y GilPérez, 1987).

Para llevar a cabo la presente investigación se elaboraron dos tipos de instrumentos: un instrumento del tipo «diferencial semántico» de 9 ítems, cuyo objetivo era determinar el Perfil socioacadémico de la física y un instrumento de escala tipo Likert de 14 ítems, cuyo objetivo era medir la actitud general de los estudiantes hacia la ciencia.

Los instrumentos del tipo «diferencial semántico» consisten en grupos de pares de adjetivos polares relacionados con el concepto que se quiere analizar, por ejemplo: útil-inutil. El estudiante marca un determinado nivel entre los dos extremos que permite apreciar su posición en referencia al tema. En nuestro estudio, el test del tipo diferencial semántico estuvo constituido por 9 ítems. De estos ítems, 5 estaban diseñados para caracterizar el Perfil académico que posee la física entre los estudiantes: fácil-difícil, interesante-aburrida, divertida-fastidiosa, comprensible-incomprensible, memorísticano memorística. Los otros 4 ítems pretendían caracterizar el Perfil social de la física: útil-inútil, segura-peligrosa, aplicable-inaplicable, beneficiosa-perjudicial. El conjunto de las respuestas a los 9 ítems se tomó como un indicador del Perfil de la percepción de la física por cada estudiante.

Se elaboró un instrumento de escala tipo Likert para determinar la actitud general del estudiante hacia la ciencia. El instrumento constaba de 14 ítems formados por enunciados que expresaban una opinión valorativa de la ciencia, por ejemplo: «las aplicaciones de la ciencia han contribuido al progreso de la humanidad», seguida de una escala que oscilaba desde «totalmente de acuerdo» (TA) hasta «totalmente en desacuerdo» (TD), con tres espacios intermedios para los que estaban parcialmente de acuerdo o en desacuerdo, y un espacio central para los neutrales o indiferentes. Un análisis de confiabilidad de este instrumento arrojó un valor para la alfa de Cronbach de 0,7084, el cual se consideró satisfactorio.

Se analizó la validez de contenido de los instrumentos mediante un panel de expertos y, en un estudio piloto, se descartaron previamente los ítems que tenían una desviación estándar inferior a 0,8 en cualquiera de los instrumentos, según las recomendaciones de Ventura (1994), ya que esto podría indicar que la discriminación de estos ítems era baja. Para la escala de actitudes tipo Likert, se descartaron en el estudio piloto aquellos ítems cuyas correlaciones con el resultado global del instrumento fuera inferior a 0,5 , con el objeto de aumentar la validez del mismo. 


\section{Análisis de los datos}

Se realizó un análisis de varianza múltiple (MANOVA), obteniéndose que los efectos de interacción entre las variables independientes eran despreciables. Al evaluar si los requerimientos para llevar a cabo los análisis de varianza eran apropiados, se encontró que las varianzas no tenían magnitudes similares (heterocedastaticidad). Por lo tanto, se descartaron los análisis de varianza (ANOVA), ya que la falta de homocedastaticidad resta potencia a los análisis paramétricos, y se decidió utilizar la $t$ de student por parejas para muestras heterocedásticas y comparar con los resultados obtenidos mediante tests no paramétricos (Kruskal-Wallis).

Se normalizó el puntaje asignado por los estudiantes a las variables Perfil y Actitud en una escala de 10 puntos. En esta escala, 5 puntos representa neutral o indiferente, los valores mayores de 5 puntos representan opiniones positivas y los valores menores de 5 puntos representan opiniones negativas. Para todos los análisis en los cuales las comparaciones pudieron ser evaluadas en cuanto a su significación estadística, se eligió un nivel alfa del $5 \%$.

\section{RESULTADOS}

\section{Evolución del Perfil de la física por nivel educativo}

En cuanto al estudio de la evolución de la variable Perfil de la física por niveles educativos (nivel 1, noveno grado de educación básica, nivel 2, primer año de educación media diversificada, y nivel 3 , segundo año de educación media diversificada) y sus correspondientes subniveles (tres trimestres para cada nivel), los resultados (Tabla I, Fig. 1) muestran que la variable Perfil evoluciona con un decrecimiento cuasilineal a lo largo del primero y del segundo nivel, mientras que en el tercer nivel muestra un comportamiento peculiar, que se manifiesta por una elevación del puntaje entre el primero y el segundo trimestre y una posterior disminución del puntaje entre el segundo y el tercer trimestre.

Tabla I

Puntaje de la variable Perfil por nivel educativo y por trimestre.

\begin{tabular}{|l|c|c|c|}
\hline Perfil & Trimestre 1 & Trimestre 2 & Trimestre 3 \\
Primer nivel & 7,2922 & 6,9973 & 6,7178 \\
Segundo nivel & 7,3608 & 7,2695 & 6,9894 \\
Tercer nivel & 7,2976 & 7,5588 & 7,2968 \\
\hline
\end{tabular}

Los resultados muestran claramente que la variable Perfil de la física experimenta una disminución cuasilineal entre los tres lapsos (trimestres) del primer nivel
Figura 1

Representación de la evolución de la variable Perfil por nivel educativo y por trimestre.

Evolución de la variable Perfil de la física

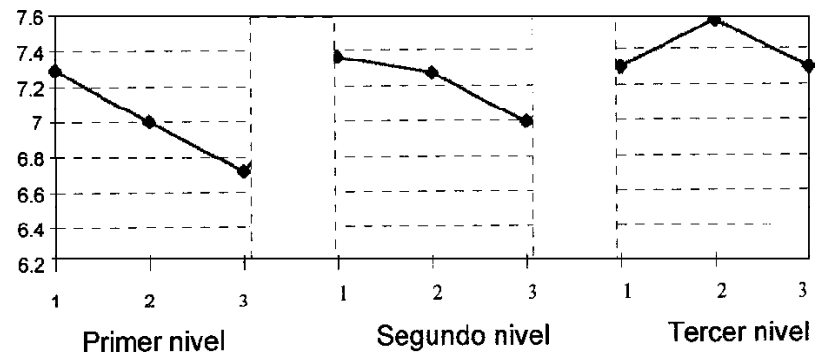

(disminución global: $\Delta x=-0,5744$, tabla I, figura 1). La evolución de las variable Perfil durante el segundo nivel también tuvo un decrecimiento cuasi lineal. La disminución de la variable Perfil de la física es debida a un deterioro tanto en el Perfil académico de la física $(\Delta x=-0,5243)$ como en el Perfil social de la misma $(x=-0,4182)$. Un análisis comparativo entre el primer trimestre y el tercer trimestre del segundo nivel mediante la $t$ de student evidencia que únicamente la variable Perfil académico es la que experimenta una disminución significativa al 5\%; la variable Perfil social, sin embargo, no experimenta disminución significativa entre ambos trimestres.

A lo largo de los tres trimestres del tercer nivel, la variable Perfil de la física experimenta un cambio casi nulo entre el primer y tercer trimestre, teniendo un ligero incremento no significativo en el puntaje entre el primero y el segundo trimestre. Este incremento podría ser debido, y esta hipótesis podría ser objeto de investigaciones posteriores, a que, en este lapso, tiene lugar en Venezuela la presentación de los exámenes de ingreso a la universidad, lo cual puede mejorar la percepción de los estudiantes acerca de la física, ya que ésta es materia obligada para el ingreso a muchas de las carreras de la universidad, mientras que otras materias no lo son. Sin embargo, durante todo el tercer nivel no existen cambios significativos de la variable Perfil, ni de las subvariables Perfil académico y Perfil social entre los diferentes trimestres.

Un análisis, mediante la $t$ de student para las variables Perfil de la física y las subvariables Perfil académico y Perfil social entre el tercer trimestre del primer nivel y el segundo trimestre del tercer nivel, arrojó diferencias significativas en todas ellas: Perfil $[t(193)=4,85$, $\mathrm{p}<0,0001]$, Perfil académico [ $t(193)=4,25, \mathrm{p}<0,0001]$, Perfil social $[t(193)=3,96, \mathrm{p}<0,0001]$. Al analizar la evolución del Perfil de la asignatura por sus componentes (Perfil académico: interesante, divertida, fácil, etc. y Perfil social: beneficiosa, útil, etc.), se determina que la evolución de ambas variables es bastante similar. Ambas experimentan un decrecimiento pronunciado a lo 
Tabla II

Evolución de la variable Perfil de física por género del estudiante.

$(\mathrm{T}=$ Trimestre, $\mathrm{F}=$ Femenino, $\mathrm{M}=$ Masculino $)$

\begin{tabular}{|l|c|c|c|c|c|c|}
\hline PERFIL & T.1-F & T.1-M & T.2-F & T.2-M & T.3-F & T.3-M \\
\hline Primer nivel & 7,1991 & 7,4108 & 6,8856 & 7,1311 & 6,6448 & 6,7863 \\
\hline Segundo nivel & 7,2191 & 7,4686 & 6,9444 & 7,5461 & 6,6842 & 7,2415 \\
\hline Tercer nivel & 6,9825 & 7,6296 & 6,9606 & 7,8916 & 6,9606 & 7,5864 \\
\hline
\end{tabular}

Representación de la evolución de la variable Perfil por nivel educativo, trimestre y género (masculino, femenino) de los estudiantes.

Evolución del Perfil de la física por género del estudiante

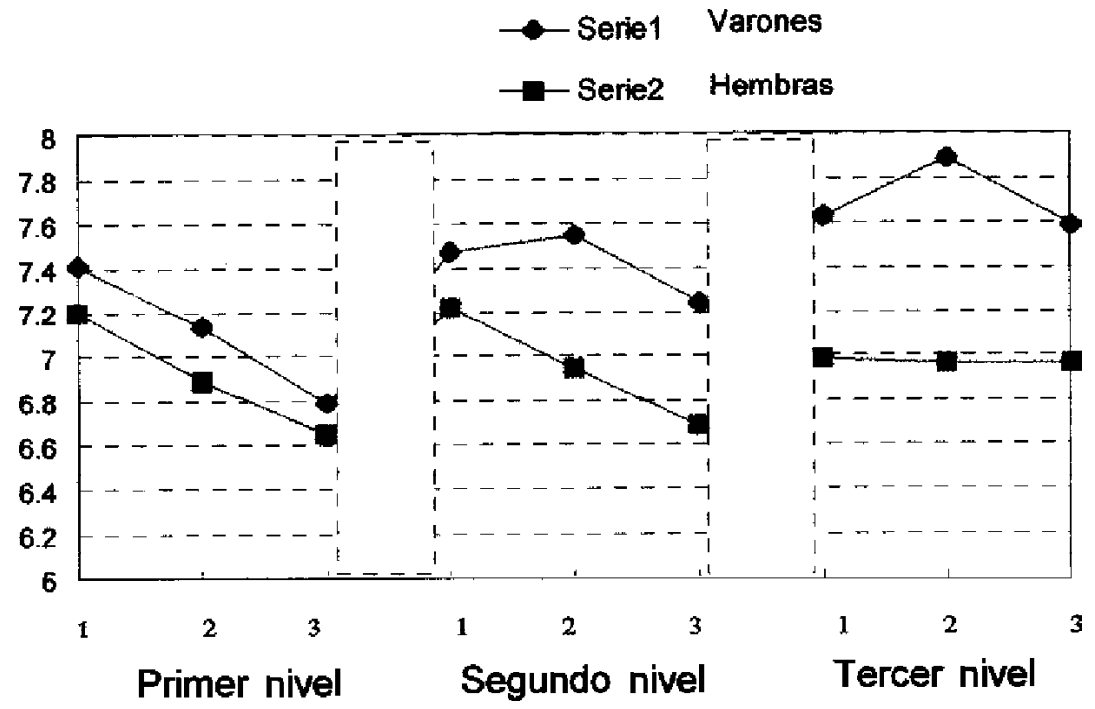

largo del año escolar y se recuperan al inicio del siguiente año. El nivel más estable es el tercer nivel, último año del bachillerato en Venezuela.

\section{Análisis de la evolución del Perfil de la física por nivel educativo y por género de los estudiantes}

La asignación de puntaje a la variable Perfil de la física resultó ser siempre superior en los varones que en las mujeres (Tabla II y Fig. 2). Al analizar los resultados de la evolución de la variable Perfil de la física según el género de los estudiantes, se observa que existe un deterioro similar para varones y para mujeres, no detectándose diferencias significativas en el deterioro de la variable Perfil durante el primer nivel $[t(133)=1,27$, $\mathrm{p}=0,208]$. En el segundo nivel, la brecha de los puntajes de la variable Perfil asignados por estudiantes varones y mujeres se hace ligeramente mayor, pero todavía no es significativa $[t(85)=1,53, \mathrm{p}=0,131]$. Es durante el tercer nivel cuando la diferencia de puntaje de la variable Perfil por género se hace significativa [ $t(67)=3,19$, $\mathrm{p}=0,002]$. Esta diferencia en las percepciones entre los varones y las mujeres se hace significativa en el tercer nivel, tanto en su aspecto de Perfil académico como en el Perfil social.

En suma, la brecha existente entre los puntajes asignados por varones y mujeres a la variable Perfil de la asignatura de física aumenta con el nivel educativo, 
Figura 3

Representación de la evolución de los puntajes de la variable Actitud hacia la ciencia con el nivel educativo y trimestre.

Evolución de la variable Actitud hacia la ciencia

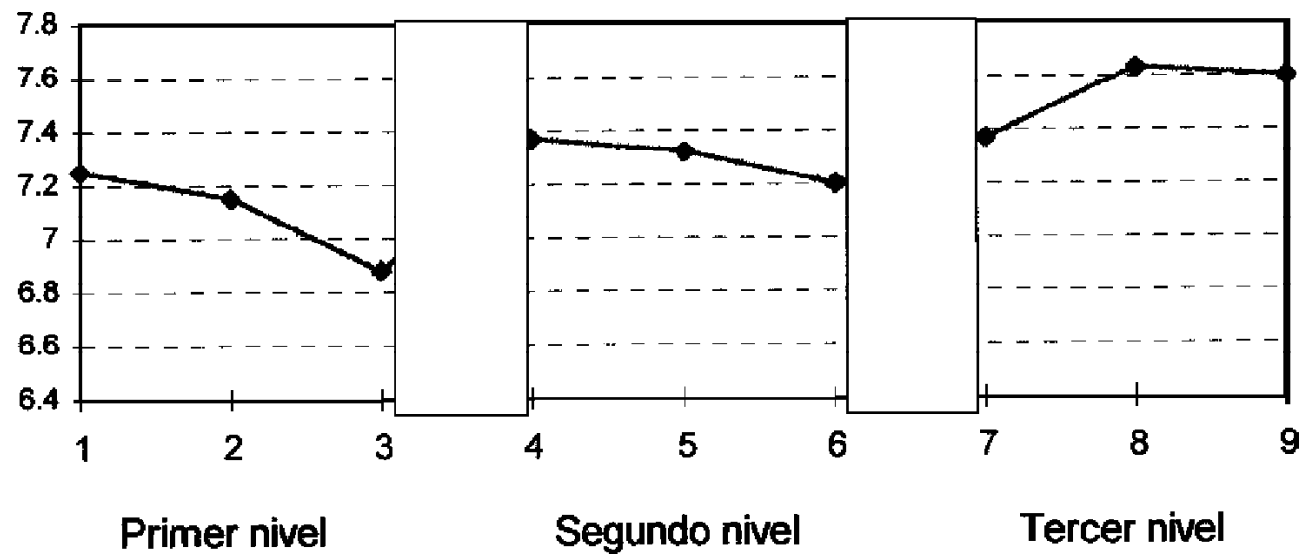

haciéndose significativa en el tercer nivel analizado (segundo año de educación media diversificada).

\section{Análisis de la evolución del Perfil de la física en función de la nota obtenida por el estudiante}

Se llevó a cabo un estudio de la evolución del Perfil de la física en función de la nota obtenida en la asignatura de física en el nivel anterior. En el caso del primer nivel, debido a que no existía nota de física del nivel anterior, ya que éste era su primer curso de física, se tomó como indicativa la nota del primer examen parcial de la asignatura.

Se detectó vinculación entre la nota de física y la percepción de la asignatura. Los estudiantes que habían obtenido notas altas (nota $>17$, en una escala de 20 puntos) mostraban percepciones más positivas que los que habían obtenido notas bajas (nota $<13$, en una escala de 20 puntos). Los resultados globales de correlación entre las variables Perfil - Nota de física muestran un coeficiente de correlación de Spearman $r=0,2346$, y que esa relación era mayor en la subvariable Perfil académico que en la subvariable Perfil social. Sin embargo, tanto los valores de correlación, como la vinculación entre la nota alta y percepción favorable de la física dejaban de ser significativos en los niveles segundo y tercero.

\section{Análisis de la evolución de la actitud de los estudiantes hacia la ciencia}

Los puntajes obtenidos mediante el instrumento de escala tipo Likert, que eran indicadores de la actitud hacia la ciencia, mostraron un patrón de evolución similar al de los puntajes asignados a la variable Perfil de la física. Es decir, la variable Actitud decrece a lo largo de los niveles primero y segundo, y se mantiene estable en el tercer nivel, experimentando un máximo en el segundo trimestre del tercer nivel (Tabla III).

Las variaciones de la variable Actitud hacia la ciencia entre los niveles y entre los trimestres son más atenuadas que las que experimenta la variable Perfil. Aunque también las actitudes decaen a lo largo del curso escolar, el puntaje asignado a esta variable se incrementa ligeramente con los niveles educativos, $\Delta x=0,3490$ entre el primer y el tercer nivel, tanto en varones $(\Delta x=0,3315)$ como en mujeres $(\Delta x=0,3628)$.

Tabla III

Evolución de la variable Actitud con el nivel educativo y trimestre.

\begin{tabular}{|c|c|c|c|}
\hline $\begin{array}{c}\text { PUNTAJE } \\
\text { ACTITUD }\end{array}$ & Trimestre 1 & Trimestre 2 & Trimestre 3 \\
\hline Primer nivel & 7,2488 & 7,1467 & 6,8791 \\
\hline Segundo nivel & 7,3681 & 7,3206 & 7,1974 \\
\hline Tercer nivel & 7,3747 & 7,6255 & 7,5978 \\
\hline
\end{tabular}

Al analizar la influencia del género (masculino, femenino) de los estudiantes en la variable Actitud hacia la ciencia, los resultados muestran que no existe diferencia significativa entre los puntajes de Actitud provenientes de los varones y de las mujeres. Por lo tanto, la evolución de la actitud general hacia la ciencia en varones y mujeres conduce a resultados mucho menos definidos que los referidos al Perfil de la física 
En el estudio de relación entre la variable Actitud hacia la ciencia con la nota de la asignatura de física, se obtuvo que la correlación entre ambas variables es significativa (coeficiente de correlación de Spearman $r=0,2429$, $p<0,0001)$.

\section{CONCLUSIONES}

La conclusión más importante que se deduce de los resultados antes expuestos, referidos a la evolución general del Perfil socioacadémico de la física y de la Actitud hacia la ciencia, es la de una erosión de ambas variables dentro del primer y del segundo nivel analizado. Estas percepciones, sin embargo, son similares al iniciar cada uno de los niveles educativos; no se obtuvo un decrecimiento de las variables Perfil y Actitud de un nivel a otro, sino únicamente un decrecimiento dentro de cada nivel.

La erosión dentro de un determinado nivel educativo (intranivel) es mayor en la variable Perfil de la física que en la variable Actitud hacia la ciencia y es ligeramente mayor en la subvariable Perfil académico, que en la subvariable Perfil social. En este sentido nuestros resultados están en consonancia con los obtenidos por Cannon y Simpson (1985), los cuales detectaron decrecimiento de actitudes positivas a lo largo del año escolar. Así pues, los estudiantes inician cada uno de los niveles educativos con similares percepciones de la asignatura de física y Actitudes hacia la ciencia. Sin embargo, en el transcurso del año escolar estas percepciones y actitudes se tornan menos positivas, posiblemente esté relacionado con un cansancio general por el año escolar. En este sentido, nuestros resultados no coinciden con los de Kelly (1986) o Macedo (1987), los cuales detectaron un claro decrecimiento de las variables actitudinales con la edad del estudiante, en edades comprendidas entre 12 y 18 años.

Al analizar las variables Perfil y Actitud, en relación con el género del estudiante, los resultados indican que los varones tienen una percepción de la física más positiva que la de las mujeres. Aunque la percepción de la física es siempre más positiva en los varones, la brecha entre varones y mujeres es inicialmente pequeña (en el primer nivel, estudiantes de unos 15 años) y aumenta progresivamente con la edad, haciéndose significativa en el tercer nivel (estudiantes de unos 17 años). Estos resulta-

\section{REFERENCIAS BIBLIOGRÁFICAS}

ARANA, J., ESCUDERO, T., GARCÉS, R. y PALACIÁN, E. (1986). El estatus socioacadémico de las matemáticas: Un punto de referencia singular. Enseñanza de las Ciencias. 4(2), pp. 129-135. dos están en consonancia con los de un estudio llevado también a cabo en Venezuela con relación a la asignatura de Matemática (Ventura, 1994) y con otras investigaciones que muestran actitudes de los varones más positivas que las de las mujeres, aunque en ocasiones la diferencia es no significativa (Haussler,1987; Arana, Escudero, Garcés y Palacián, 1986). Sin embargo, en algunos de ellos (Hofstein, Ben-Zvi y Samuel, 1976; Haussler, 1987), la brecha existente entre la percepción de los varones y las mujeres evolucionaba en el sentido de disminuir con la edad en lugar de aumentar.

La vinculación entre la nota de la asignatura de Física con el Perfil de la física y la Actitud hacia la ciencia presenta en nuestro estudio una relación positiva y significativa. Los estudiantes que obtienen notas altas tienen una mejor percepción de la física y una actitud más favorable hacia la ciencia. Esta asociación, sin embargo, disminuye con el nivel educativo y, por tanto, con la edad, lo cual parece indicar una cierta estabilización de las valoraciones de los alumnos con independencia de su rendimiento.

\section{LIMITACIONES DEL ESTUDIO}

Todo estudio conlleva limitaciones de diferente tipo, las cuales restringen la extensión de sus resultados, incluso dentro de la misma población. Entre las limitaciones del presente estudio destacamos las siguientes:

1) El estudio se llevó a cabo en diferentes niveles educativos pertenecientes a una única institución de educación privada (nivel socioeconómico alto). Dado que Venezuela es un país con una estratificación social muy marcada, sus resultados son difícilmente extrapolables a grupos de estudiantes que pertenezcan a otros niveles socioeconómicos. Consideramos que sería deseable extender este estudio a otros niveles de la población para comparar sus resultados.

2) Los instrumentos que pretenden reflejar actitudes y opiniones son siempre menos confiables que los instrumentos de conocimientos. Por lo tanto, los resultados podrían variar sensiblemente al ser medidos con otro tipo de instrumentos. Consideramos fundamental desarrollar en nuestra lengua instrumentos válidos y confiables para la determinación de intereses y actitudes relativos a diferentes disciplinas científicas.
CANNON, R.K. y SIMPSON, R.D. (1985). Relationship among attitude, motivation, and achievement of ability grouped, seventh-grade life science students. Science Education, 69(2), pp. 121-138. 
ESCUDERO, T. y LACASTA, E. (1984). Las actitudes científicas de los futuros maestros en relación con sus conocimientos. Enseñanza de las Ciencias, 2(3), pp. 175-180.

FRASER-ABDER, P. y SHRIGLEY, R.L.A. (1980). Status study of the science attitudes of elementary school teachers in Trinidad and Tobago. Science Education, 64(5), pp. 637644.

FRASER, B.J. (1977). Selection and validation of attitude scales for curriculum evaluation. Science Education, 61(3), pp. 317-329.

FRASER, B.J. (1982). How strongly are attitude and achievement related? School Science Review, 63, pp. 557-559.

GABEL, D. (1981). Attitudes toward science and science teaching of undergraduates according to major and number of science courses taken, and the effect of the two courses. School Science and Mathematics, 81, pp.70-76.

GARDNER, P.L. (1975). Attitudes to Science: A review. Studies in Science Education, 2, pp. 1-41.

GOGOLIN, L. y SWARTZ, F. (1992). A quantitative and qualitative inquiry into attitudes toward science of nonscience college students. Journal of Research in Science Teaching, 29(5), pp. 487-504.

HADDEN, R.A. y JOHNSTONE, A.H. (1983). Secondary school pupils'attitudes to Science: the year of erosion. European Journal of Science Education, 5(3), pp. 309-317.

HAMILTON, M.A.(1982). Jamaican Students'attitude to science as it relates to achievement in external examinations. Science Education, 66(2), pp. 155-169.

HASSAN, O. (1985). An investigation into factors affecting attitudes toward Science of Secondary School students in Jordan. Science Education, 69(1), pp. 3-18.

HAUSSLER, P. (1987). Measuring students' interest in physicsdesign and results of a cross-sectional study in the Federal Republic of Germany. International Journal of Science Education, 9(1), pp. 79-92.

HOFSTEIN, A., BEN-ZVI, R. y SAMUEL, D. (1976). The measurement of the interest in, and attitudes to, laboratory work amongst Israeli High School Chemistry Students. Science Education, 60(3), pp. 401-411.

HOFSTEIN, A., BEN-ZVI, R., SAMUEL, D. y TAMIR, P. (1977). Attitudes of Israeli High-School students toward Chemistry and Physics: a comparative study. Science Education, 62(2), pp. 259-268.

KELLY, A. (1986). The development of girls and boys attitudes to science: A longitudinal study. European Journal of Science Education, 8(4), pp. 399-412.

KOBALLA, T.R. (1992). Persuasion and attitude change in Science Education. Journal of Research in Science Teaching, 29(1), pp. 63-80.

KOZLOW, M.J. y NAY, M.A. (1979). An approach to measuring scientific attitudes. Science Education, 58(2), pp. 147-172.

KUHN, D.J. (1979). Study of the attitudes of secondary school students toward energy-related issues. Science Education, 63(5), pp. 609-620.

MACEDO, B. (1987). Actitudes de los educandos frente al aprendizaje de la química: implicancias y consecuencias.
Resúmenes del Segundo Congreso Internacional de Didáctica de las Ciencias y de las Matemáticas, p. 254. Valencia.

MALONE, M.R. y FLEMING, M.L. (1983). The relationship of student characteristics and student performance as viewed by meta-analysis research. Journal of Research in Science Teaching, 20, pp. 481-485.

MONCADA, P. y SEBASTIÁ, J.M. (1986). Actitudes hacia la física de estudiantes venezolanos: Una primera aproximación. Jornadas Nacionales de Enseñanza de la Física. CENAMEC. Caracas. Venezuela.

MORENO, M.D. y GIL, D. (1987). La medida de las actitudes de los estudiantes de BUP hacia la física. Resúmenes del Segundo Congreso Internacional de Didáctica de las Ciencias y de las Matemáticas, pp. 268-269.Valencia.

OLIVER, J.S. y SIMPSON, R.D. (1988). Influences of attitude toward science achievement, motivation and science self concept on achievement in Science: a longitudinal study. Science Education, 72(2), pp. 143-155.

OSGOOD, C.E. (1969). Semantic differential technique. Chicago: Aldine.

PETERSON, R.W. y CARLSON, G.R. (1979). A summary of research in science education, 1977. Science Education, 59, pp. 207-210.

SADAVA, D. (1976). Attitudes toward science of nonscience major undergraduates compared with the general public and effect of a science course. Journal of Research in Science Teaching, 13, pp. 78-84

SCHIBECI, R. A. (1982). Measuring students attitudes: semantic differential or Likert instruments? Science Education, 66(4), pp. 565-570.

SCHIBECI, R.A. (1984). Attitudes to Science: an Update. Studies in Science Education, 11, pp. 26-59.

SCHIBECI, R.A. (1986). Images of science, scientist and science education. Science Education, 70(2), pp. 139-149.

SCHIBECI, R.A. (1989). Home, school and peer influences on student attitudes and achievement in science. Science Education, 73(1), pp. 13-24.

SCHIBECI, R.A. y RILEY, J.P. (1986). Influence of students' background and perceptions on science attitudes and achievement. Journal of Research in Science Teaching, 23(3), pp. 177-187.

SHRIGLEY, R.L. (1983). The attitude concept and science teaching. Science Education, 67(4), pp. 425-442.

SOLOMON, J. (1987). Social influences on the construction of pupil's understanding of science. Studies on Science Education, 14 , pp. 63-82.

VENTURA, J.C. (1994).Construcción de una escala de actitudes hacia la matemática. Resúmenes del VII Seminario Nacional de Investigación Educativa. p. 44. Caracas. Venezuela.

YAGER, R.E. y PENICK, J.E. (1984). What students say about science teaching and science teachers. Science Education, 68(2), pp. 143-152.

YAGER, R.E. y PENICK, J.E. (1986). Perceptions of four age groups toward science classes, teachers, and the value of Science. Science Education, 70(4), pp. 355-363. 